\title{
L'anglais de spécialité en LEA : entre proximité et distance, un nouvel équilibre à construire
}

\section{Marie-Françoise Narcy-Combes}

\section{(2) OpenEdition}

\section{Journals}

Édition électronique

URL : http://journals.openedition.org/asp/396

DOI : $10.4000 /$ asp.396

ISBN : 978-2-8218-0406-7

ISSN : 2108-6354

Éditeur

Groupe d'étude et de recherche en anglais de spécialité

Édition imprimée

Date de publication : 1 novembre 2008

Pagination : 129-140

ISSN : 1246-8185

\section{Référence électronique}

Marie-Françoise Narcy-Combes, «L'anglais de spécialité en LEA : entre proximité et distance, un nouvel équilibre à construire », ASp [En ligne], 53-54 | 2008, mis en ligne le 01 novembre 2011, consulté le 19 avril 2019. URL : http://journals.openedition.org/asp/396 ; DOI : 10.4000/asp.396

Ce document a été généré automatiquement le 19 avril 2019

Tous droits réservés 


\title{
L'anglais de spécialité en LEA : entre proximité et distance, un nouvel équilibre à construire
}

\author{
Marie-Françoise Narcy-Combes
}

\section{Introduction}

1 Lorsque l'université de Nantes a recruté un maitre de conférences pour Langues Étrangères Appliquées (LEA) en 2000, la mission qui lui était attribuée était de réfléchir à l'organisation de l'enseignement de l'anglais dans la filière et de le restructurer. La didacticienne et spécialiste en anglais des affaires que j'étais alors avait traduit que son travail serait de s'employer à réduire la distance entre ce que nous disent les théories didactiques et la réalité des pratiques de terrain. L'entreprise était d'envergure et a donné lieu à plusieurs publications sur le sujet. En six années de travail de terrain en LEA, mes collègues et moi avons progressivement modifié le paysage en accompagnant la réflexion sur le contexte d'enseignement, le public et les objectifs visés, les contenus d'enseignement, les tâches et les dispositifs de recherche-action dont nous allons présenter ici l'aboutissement.

2 Mon positionnement en tant que chercheure, à travers le prisme de vingt ans d'expérience d'enseignement dans le secteur LANSAD, dont les huit dernières années en LEA accompagnées d'activités de recherche dans ce domaine, est que, bien que l'anglais de spécialité soit par définition la langue enseignée en LEA, et en cela on est très proche de la problématique LANSAD, le contexte dans lequel cet enseignement intervient présente des différences notables et impose une transposition didactique des recherches en langue de spécialité qui tienne compte non seulement des contraintes institutionnelles, mais également des paramètres socio-économiques qui conditionnent le recrutement dans la filière et les débouchés potentiels. Nous ferons le point dans un premier temps sur les références théoriques qui sous-tendent notre action sur le terrain. 
Dans un second temps, c'est le terrain lui-même que nous tenterons de définir, en mettant en évidence les liens entre théorie et pratique. Selon la thématique retenue pour le congrès du GERAS 2008, nous verrons comment la tâche a permis d'établir des ponts entre " distance et proximité ", deux concepts non antinomiques. Cette réflexion a abouti au projet actuel de mise en place d'un dispositif hybride d'enseignement/apprentissage de l'anglais en LEA que nous présenterons, qui allie proximité et distance et les réconcilie. Nous conclurons sur l'opportunité de développer une collaboration et des échanges concernant l'enseignement / apprentissage de l'anglais dans le contexte spécifique de LEA.

\section{Fondements théoriques}

3 Notre cadrage théorique relève pleinement du champ de la didactique des langues, qui se réclame des sciences humaines. Dans ce cadre, la méthodologie joue un rôle déterminant au service de la rigueur scientifique. Il conviendra donc de distinguer entre expérience pédagogique et expertise didactique. Pour le didacticien, l'innovation pédagogique ne peut se faire sans analyse didactique sous peine de rester non scientifique. En effet, s'il est possible à tout enseignant de se lancer dans une innovation au sein de sa classe, celle-ci n'est pas envisageable s'il n'a pas réfléchi aux raisons pour lesquelles elle s'avère nécessaire, aux problèmes posés, à la mise en place de cette innovation, aux moyens d'en évaluer l'efficacité, etc. À partir du moment où cette réflexion existe, on est bien dans le champ de la didactique des langues. Si elle n'existe pas, un problème se pose. À l'échelle d'une formation universitaire portant sur des grands nombres, c'est la rigueur méthodologique qui garantira la validité scientifique des dispositifs mis en place.

Les effets de contexte devront être pris en compte lors de la mise en place des dispositifs d'enseignement/apprentissage : ce n'est pas la même chose que d'enseigner la langue des affaires dans une grande école ou même un IUT, et d'enseigner en LEA, ou dans le secteur LANSAD, pas plus qu'il n'est possible à l'université de transférer les pratiques de LLCE à LEA sans réflexion didactique. ${ }^{1}$ La plupart des problèmes rencontrés dans la filière LEA lors de mon arrivée tenaient essentiellement à ce que le contexte n'avait pas été suffisamment analysé et que les cours en vigueur en LLCE avaient été transposés à LEA sans adaptation suffisante. De même on ne peut pas déterminer de politique linguistique sans prendre en compte les réalités sociolinguistiques du contexte (Kachru in Seidlhoffer 2003). Il n'existe donc pas de réponse univoque à la question de l'efficacité des dispositifs d'enseignement/apprentissage des langues.

5 Enfin, il sera nécessaire de prendre en compte les théories en acquisition des langues. Nous postulons en effet à la suite, entre autres, de Chapelle (2003) et de Jordan (2004) que la mesure d'une action ou d'un dispositif d'apprentissage doit se faire en termes d'une théorie d'acquisition de L2.

6 Nous avons donc commencé par prendre la mesure de l'existant, par rechercher quelles étaient les pratiques et les raisons de ces pratiques pour en faire l'analyse historique, factuelle et sociologique. C'est une position qui ne va pas de soi. Elle est difficile à tenir car le terrain est mouvant, particulier et que la progression est semée d'embûches. La France étant une culture de l'implicite, le nouvel arrivant dans une organisation doit d'abord en décoder le fonctionnement, sans que l'aide nécessaire lui soit apportée, ce qui est source de lenteur, d'inefficacité, voire d'erreurs d'interprétation et de conduite qui ne favorisent pas l'intégration. Le phénomène est encore aggravé par l'organisation 
bureaucratique de l'institution. Il faut ajouter à cela le caractère conservateur des représentations, source de résistance passive ou active de la part des collègues et des étudiants à l'innovation proposée. Pour que l'évolution soit possible, il sera nécessaire que le contexte social, culturel et éducationnel s'y prête. Ces considérations concernent aussi le chercheur, c'est pourquoi comme le rappellent Morin (1977) et Elias (1993) le chercheur doit être réintégré dans sa recherche. ${ }^{2}$ Lors de ma prise de fonctions, l'enseignement de l'anglais en LEA était désorganisé. Les enseignants venaient y faire cours en traînant les pieds parce qu'on les avait contraints à assurer un minimum de vingt-quatre heures de cours en LEA. On comptait peu d'enseignants permanents nommés pour LEA. Les résistances étaient fortes comme le montre cette réflexion d'une enseignante d'anglais, pourtant à temps complet en LEA: "Avant personne ne s'intéressait à LEA, alors on pouvait faire ce qu'on voulait ».

7 L'analyse des besoins constitue l'étape suivante. Elle ne saurait se faire sans prendre la mesure des objectifs visés et de la réalité du terrain. Dans le cas des étudiants de LEA il importe de savoir qui les emploie et sur quelles bases, et s'il existe un créneau qui serait plus porteur pour assurer des débouchés professionnels. Force est de constater sur ce point une certaine tendance à servir les intérêts de l'institution et des personnels, et à justifier les choix par une rationalisation a posteriori. Les objectifs d'enseignement/ apprentissage de l'anglais pour les étudiants seront déterminés en fonction de ces analyses et des apports des théories puis le dispositif pourra être mis en place pour leur permettre de s'approprier le savoir dont ils ont besoin. Cela ne peut consister en un apport de recettes et de principes à apprendre par cœur qui ne peuvent permettre de gérer la diversité : nous postulons qu'il ne suffit pas de transmettre des contenus, mais qu'il est nécessaire de surcroît d'offrir un cadre théorique aux étudiants qui puisse leur permettre de développer leurs capacités en toute autonomie. Dans le cas des enseignants, qui posent un problème de recrutement propre à cette filière ${ }^{3}$, il sera nécessaire de s'interroger sur les outils nécessaires pour faire face à la réalité des situations de terrain et sur la façon dont on pourra leur offrir les moyens de trouver des réponses spécifiques à la diversité de leurs situations. De plus, nous nous trouvons dans la mouvance : ainsi, selon Elias (1993) l'homme est un processus défini par la société de son époque : il est multiple ou, selon Lahire (2001), pluriel.

Il sera enfin nécessaire de mesurer les effets du dispositif qui devra être évalué, ainsi que les acteurs. C'est là un point important, or force est de constater qu'il existe un manque crucial dans ce domaine alors même que l'absence de mesure et d'évaluation des dispositifs, des étudiants et des enseignants, parce qu'elle induit l'absence de nécessité de rendre des comptes ainsi que l'exprime avec peut-être plus de pertinence le concept britannique de accountability, risque de conduire à des dérives et/ou à la déresponsabilisation. Les récentes mesures gouvernementales visent à pallier cette lacune. On peut cependant déplorer qu'une fois de plus cette évaluation nécessaire ait été parachutée sans proposition de formation et d'explication. Ces principes théoriques prennent leurs sens dans leur application au contexte où ils opèrent et auxquels ils doivent être adaptés.

\section{Le Contexte LEA}

9 Les points communs entre LEA et LANSAD sont nombreux, néanmoins, nous l'avons dit plus haut, il existe des spécificités contextuelles qui ne sauraient être négligées. Les 
processus à l'œuvre en LANSAD sont transférables à d'autres domaines, en LEA notamment, mais c'est la recherche qui procure le recul nécessaire pour le faire. Ainsi, la théorie s'ancre-t-elle dans la pratique pour accomplir une mission d'utilité sociale qui les rapproche.

Par ailleurs, l'anglais des affaires enseigné en LEA est une des branches de l'anglais de spécialité dont l'objectif est de permettre la communication au sein d'une communauté discursive donnée à travers une langue et un discours commun et la maîtrise de genres reconnus dans et par la communauté. Il s'agit donc d'un enseignement professionnalisant pour lequel se retrouve l'objectif social associé à la notion de rentabilité de la formation, un concept directement lié à la formation en entreprise.

11 La filière LEA présente des pesanteurs qui sont aussi des richesses. La lourdeur des grands nombres, un des problèmes les plus difficiles à gérer, est aussi un signe de succès comme le montre le nombre de participants aux journées portes ouvertes de l'Université de Nantes. Le plurilinguisme, comme la pluridisciplinarité, sont des atouts qui permettent flexibilité et adaptabilité, mais ces aspects ont aussi été critiqués et on a pu parler de connaissances superficielles et de dispersion, faisant ainsi peser le soupçon sur le niveau des étudiants dans chacune des spécialités. Quant à l'ouverture sur l'entreprise, elle a donné lieu à des accusations d'ancillarité et de manque d'indépendance contraires à l'esprit universitaire. Cependant, LEA offre plus de chances de trouver un emploi que d'autres filières littéraires. En effet, si l'on considère simplement le taux d'embauche à la sortie des deux Masters professionnels en LEA de notre université, celui-ci est proche de $100 \%$, ce qui laisse bien loin derrière les formations CAPES ou Agrégation.

Dans le contexte spécifique de cette étude, le travail de recherche didactique a consisté à réduire progressivement sur la période qui s'étend de 2000 à 2008 la distance existant entre les pratiques de terrain et ce que nous disent les théories en acquisition de L2, et à donner à l'équipe des enseignants d'anglais dans la filière les moyens pour le faire. C'est ainsi qu'en un peu plus de six ans on est passé d'un faible investissement enseignant, dû essentiellement à l'image peu valorisante de LEA, à l'organisation d'équipes investies qui travaillent ensemble grâce, entre autres, à une politique volontariste de recrutement. En effet, en 2000 la filière en anglais ne comptait, en tant qu'enseignants titulaires permanents nommés pour LEA, qu'un Maître de conférences, trois professeurs agrégés et un PAST, un traducteur professionnel qui faisait la moitié de son service en enseignement. En 2008 elle est forte d'un poste de professeur, cinq maîtres de conférences, et cinq professeurs agrégés pour près de six cent étudiants en première année, des effectifs qui restent malgré ces avancées bien en deçà de ceux de la filière traditionnelle de LLCE. À titre de comparaison, pour un effectif total de 273 étudiants en première année, on compte six professeurs, seize maîtres de conférences et un professeur agrégé. Les résultats montraient une forte déperdition des effectifs de L1 à M1 due en partie à l'insuffisance des résultats, en particulier en anglais. L'organisation en cours magistraux, travaux dirigés et travaux pratiques, calquée sur celle des LLCE, paraissait inadaptée à une formation professionnalisante dont le nombre total d'étudiants équivaut à celui de toutes les formations LLCE réunies. Aujourd'hui nous sommes arrivés à une cohérence des contenus de formation L1 à M2 autour du commerce international. Les résultats en anglais langue de spécialité et traduction sont en hausse et nous travaillons à la mise en place d'un dispositif hybride d'enseignement/ apprentissage de l'anglais à l'aide de la plate-forme d'enseignement de l'université pour la rentrée 2008. 


\section{De l'analyse des besoins à la mise en place d'un dispositif adapté}

13 Les différentes études menées au cours des années nous ont conduits à travailler la cohérence des contenus en lien avec leur avenir professionnel: comprendre les spécificités de la langue des affaires en termes de genre discursif, registre de langue, lexique spécifique et collocations, mais également développer des compétences professionnelles comme être capable de mener à bien un projet en équipe, savoir mener une recherche documentaire et en rendre compte dans une ou plusieurs langues, être capable d'autonomie et savoir prendre des responsabilités. L'approche par les tâches nous est apparue le mieux à même de réduire la distance entre idéal théorique et réalité du terrain. Si l'on se fonde sur les théories en acquisition des langues, nous pouvons postuler que les apprenants seront prêts à s'impliquer si on leur propose une tâche en lien avec leur environnement professionnel (Ellis 2003). En effet, si les tâches proposées sont réalistes en termes de discours et de contenus, si elles ont une authenticité socioculturelle, leur légitimité sera plus grande aux yeux des apprenants. Par ailleurs, l'étude des processus d'apprentissage nous indique que la production langagière résulte d'un double processus par règle et par instances, et que toutes les productions sont nativisées, c'est-à-dire qu'elles sont le résultat d'une analyse de L2 que l'apprenant effectue en fonction de critères personnels comme par exemple ce qu'il sait ou croit savoir sur L2. Nous avons également intégré dans cette réflexion le rôle et la place du repérage (noticing) et du traitement du sens en profondeur (deep processing) qui jouent un rôle déterminant dans l'acquisition linguistique (Narcy-Combes J.-P. 2005, Robinson 2003) et pris en compte les théories psycholinguistiques qui indiquent que si l'apprentissage explicite facilite le repérage, il ne conduit pas nécessairement à une connaissance implicite, du moins pas directement. On cherchera donc à proposer aux étudiants de LEA des tâches complexes comme des simulations, des cyber-enquêtes, mais aussi des tâches plus directement universitaires telles que des contractions de textes, des résumés, des présentations orales, des comptes rendus, etc. En fonction des écarts entre les productions des apprenants et les productions attendues, l'enseignant les dirigera vers des micro-tâches individuelles d'entraînement et/ou d'explicitation à réaliser en centre de ressources virtuel ou non. En fonction de quoi on peut raisonnablement émettre l'hypothèse que parce que les étudiants seront conduits par la nature de la tâche à repérer des éléments signifiants et à agir au niveau du sens dans un environnement réaliste et authentique, il y aura acquisition.

En fonction de ces présupposés théoriques et méthodologiques nous avons déterminé, pour chaque niveau de la formation en LEA, des objectifs à atteindre et une tâche ou une série de tâches destinées à permettre la mise en place des savoirs et savoir-faire définis par ces objectifs, par exemple la simulation de la mise en place d'un projet d'entreprise en M1, une nouvelle approche de la traduction davantage centrée sur les réalités de la vie professionnelle en L2 (Muller \& Schmidt 2007). La mise en place des dispositifs s'appuie sur des données issues du cadre théorique sur le plan nomothétique, on est alors dans la distance, et il est adapté au contexte sur le plan idiographique, on est alors dans la proximité. Il est validé selon un protocole d'évaluation qui inclut la progression et la satisfaction des étudiants mesurées par questionnaire, l'observation directe, un pré-test et un post-test, les résultats à l'examen, et des entretiens (pour un exemple de résultats 
comparatifs à l'examen en L2, voir annexe 1). Ceci peut être réalisé grâce à la contribution d'étudiants en Master 1 et 2 de didactique des langues.

Forts des résultats obtenus au cours des années (Narcy-Combes M.-F. 2003, 2005, 2008a, 2008b, Narcy-Combes M.-F. \& Narcy-Combes J.-P. 2007) nous avons décidé d'aller plus loin et de proposer la mise en place d'un dispositif hybride d'enseignement/apprentissage en langue de spécialité et traduction de L1 à M1 utilisant la plate-forme d'enseignement de l'université opérationnelle depuis septembre 2006. Ce dispositif combine enseignement à distance et en présentiel et s'appuie sur une combinaison de micro- tâches et de macrotâches comme outils de formation et de résolution de problèmes.

Les avantages d'un tel dispositif sur le plan théorique se justifient par l'inefficacité démontrée des systèmes transmissifs pour l'acquisition des langues; les systèmes hybrides a contrario facilitent l'activité de l'apprenant (Aymard 2005). Ils permettent l'individualisation des parcours et facilitent la mise en place d'un suivi personnalisé (Vigotsky 1997, Bruner 2000). Sur le plan pratique, ils rendent la gestion des grands nombres plus aisée et le travail oral peut être renforcé. Enfin, parce que l'apprenant doit prendre en charge une partie de son apprentissage, ils rendent possible le développement de l'autonomie et la responsabilisation.

Les limites sont liées au risque d'abandon élevé dû au sentiment d'isolement que ressentent les apprenants dans ce type de système et qu'il faut prévoir de pallier grâce à des regroupements et à des tâches à réaliser en équipe. Dans un premier temps, l'apprenant sera accompagné pour lui permettre d'utiliser la plate-forme et de se repérer dans les diverses composantes du dispositif, grâce à l'utilisation du tutorat. Une aide disciplinaire lui sera offerte, fournie par un expert dans le domaine d'apprentissage choisi, ainsi qu'une aide cognitive et métacognitive sous la forme d'un accompagnement dans le choix des stratégies d'apprentissage et l'acquisition de l'autonomie. Comme le soulignait Christine Vaillant lors du congrès de l'APLIUT en juin 2007, il conviendra également de prévoir l'accompagnement des enseignants susceptibles d'intervenir dans cette formation pour leur fournir le cadre théorique et pratique qui les mettra à même de choisir les outils qui leur conviendront le mieux, voire de développer leurs propres ressources multimédia, mais aussi d'acquérir le socle minimum de connaissances en technologies de l'information et de la communication (TIC) pour la conception et la création de ressources.

18 L'utilisation de la plate-forme de l'université nous permettra, dès la rentrée 2008 si tout se passe comme prévu, de concilier distance et proximité, avec l'objectif que la distance permette justement de réduire cette dernière, celle qui s'établit nécessairement dans la relation pédagogique lorsque le nombre d'étudiants est supérieur à vingt, celle également qui sépare les productions des étudiants des attentes des enseignants de langue. Le public visé est celui des étudiants de LEA de L1 à M1.Les instances de l'université ne nous ont pas permis d'étendre le dispositif comme nous le souhaitions, et il ne sera mis en place en 2008 qu'au niveau L1, au second semestre, à titre expérimental.

19 En ce qui concerne l'enseignement à distance, tout ce qui relève de la distance sera mis sur la plate-forme : ce qui relève des contenus, c'est-à-dire les cours, les ressources, les liens Internet, ce qui relève de la construction des savoirs et savoir-faire, c'est-à-dire les tâches à accomplir par les étudiants selon un calendrier établi, ainsi que des dispositifs de communication tels que des forums de demande d'aide, de discussion et d'instructions et de consignes, et enfin ce qui relève de l'évaluation. En ce qui concerne l'organisation du travail, les étudiants constituent des groupes de quatre au maximum et réalisent dix 
tâches chaque semestre selon un calendrier défini en commun (synthèse de documents, compte rendu d'une langue à l'autre, études de cas, projets, réflexion méta, etc.). Chaque enseignant gère un groupe de 45 étudiants.

Pour l'enseignement « de proximité » (le présentiel) nous avons prévu des regroupements de quinze étudiants, en comptant que chaque groupe pourra en moyenne rencontrer un enseignant pendant une heure chaque semaine. Ces regroupements seront consacrés au travail oral et à la rétroaction ou information critique (Narcy-Combes J.-P. \& Walsky 2004) sous forme de conseils, de suggestion de corrigés et d'autocorrection (Duda 2001). Une partie de ce retour sur les productions des étudiants se fera à distance, par l'intermédiaire de la plate-forme, ce qui sera rendu possible et plus facile grâce au travail en équipe des enseignants et à la constitution d'un fichier maitre permettant la mise en commun des informations sur les erreurs les plus fréquentes et les conseils les plus souvent donnés : un simple copier-coller devrait alors être suffisant dans la plupart des cas pour guider des apprenants francophones à développer leur interlangue de façon plus efficace.

21 La validation combine le contrôle continu et l'examen, à 50 \% pour chacun. Le contrôle continu s'effectue sur la base du contrat pédagogique. Les dix tâches doivent être correctement réalisées et rendues à l'enseignant obligatoirement. Une tâche manquante donne lieu à une perte de $25 \%$ des points, deux tâches manquantes font perdre $50 \%$, et au-delà aucun point n'est comptabilisé pour le contrôle continu. Une tâche n'est pas considérée comme accomplie si l'autocorrection n'a pas été faite. L'examen final écrit de trois heures sanctionne un niveau individuel. On trouvera en annexe 2 la proposition de programme qui découle de ces réflexions.

\section{Discussion et conclusion}

Le chemin qui reste à parcourir avant de finaliser la mise en place de ce projet est encore long et nous n'avons pas fini d'avancer. Nous avons besoin de communiquer plus largement au sein même de l'université pour remporter l'adhésion car les résistances inhérentes à toute innovation existent. Il sera également nécessaire d'accompagner l'expérimentation d'un dispositif de recherche-action et de se doter d'outils de recueil et d'analyse de données pour mieux comprendre un contexte mouvant, ce qui implique le travail en commun d'une équipe de chercheurs en didactique des langues. Celle-ci se construit et se renforce et il est possible aujourd'hui de compter sur une collaboration solide dans ce domaine, ce qui devrait faciliter la tâche. Nous avons également constitué un groupe de réflexion pour la mise en place du dispositif. Il sera ouvert aux nouveaux arrivants pour que les différents intervenants puissent s'approprier le système et se former à de nouvelles modalités pédagogiques. Nous avons également prévu d'accompagner les enseignants dans l'utilisation de l'outil informatique grâce à la collaboration active des techniciens.

Notre hypothèse est que ce dispositif est mieux adapté aux besoins et aux compétences du public ciblé. Il permettra de mettre en place une structure collaborative et de réduire la distance qui sépare savoirs théoriques et représentations des différents acteurs dans le souci d'accroître l'efficacité de la formation proposée. Ce sont des réalisations concrètes comme celle-ci qui peuvent assurer le renforcement de la crédibilité et du rayonnement de la filière LEA, parce qu'elles permettent de montrer comment on peut fédérer des énergies, susciter l'intérêt des collègues et les conduire à se motiver pour un projet, 
emporter l'adhésion des instances institutionnelles, et nous l'espérons, à terme, améliorer la réussite des étudiants à l'université.

Ceci nous paraît indissociable du développement de la réflexion didactique et disciplinaire en LEA. En effet, lorsqu'on enseigne une langue, on lui confère une légitimité politique, sociale, culturelle. Chaque nouvelle personne qui parle cette langue contribue à étendre son influence. Les conséquences humaines, écologiques et économiques sont considérables d'où la nécessité de développer la responsabilité épistémologique des différents acteurs, enseignants et étudiants, et d'abord chez soi-même, c'est-à-dire garantir la validité des savoirs concernant les faits, les raisonnements, les méthodes, les concepts propres à un champ donné en se gardant de tout dogmatisme, comme de tout relativisme radical, ce qui est d'autant plus difficile lorsqu'il s'agit d'œuvrer dans un champ de références instable et donc de gérer l'incertitude, mais de ce fait d'autant plus nécessaire.

\section{BIBLIOGRAPHIE}

Aymard, V. 2005. « Environnements virtuels et didactique des langues, quelle réalité ? ». Thèse de doctorat, Université Paris 3.

Bruner, J. 2000. Culture et modes de pensée : l'esprit humain dans ses œuvres. Paris : Retz.

Chapelle, C. A. 2003. English Language Learning and Technology. Amsterdam/Philadelphie : John Benjamins.

Duda, R. 2001. « Apprentissage autodirigé : le rôle du conseiller ». Le Français dans le monde 318, 34-36

Elias, N. 1993. Engagement et distanciation. Paris : Fayard.

Ellis, R. 2003. Task-based Language Learning and Teaching. Oxford : Oxford University Press.

Jordan, G. 2004. Theory Construction in Second Language Acquisition. Amsterdam : John Benjamins.

Lahire, B. 2001. L'homme pluriel, les ressorts de l'action. Paris : Nathan.

Morin, E. 1977. La nature de la nature. Paris : Seuil.

Muller, E et B. Schmidt. 2007. Traduction anglaise : maîtriser l'outil Internet. Paris : PUF.

Narcy-Combes, J.-P. 2005. Didactique des langues et TIC : vers une recherche-action responsable. Paris : Ophrys

Narcy-Combes M.-F. 2003. « La communication interculturelle en anglais des affaires : transfert ou conflit d'interprétation?». ASp 39-40, 119-129.

Narcy-Combes M.-F. 2005. « Quelle recherche didactique pour la filière LEA ? ». ASp 47-48, 155-163.

Narcy-Combes M.-F. 2008a. « Conflits de représentations et adaptation des dispositifs d'enseignement/apprentissage ». Les Cahiers de l'APLIUT 27/1, 33-50. 
Narcy-Combes M.-F. 2008b. « Les plates-formes : une réponse aux problèmes d'enseignement/ apprentissage des langues dans le contexte universitaire ? ». Les Cahiers de l'APLIUT 27/2, 92-107. Narcy-Combes M.-F. et J.-P. Narcy-Combes. 2007. « La tâche, réponse à des problèmes spécifiques dans le contexte universitaire français ». Le Français dans le Monde « Langueet travail », 73-86 Narcy-Combes, J.-P. et J. Walsky. 2004. « Le concept de tâche soumis au crible de nouvelles questions ». Les Cahiers de l'APLIUT 23/1, 27-24.

Seildhoffer, B. 2003. Controversies in Applied Linguistics. Oxford : Oxford University Press. Robinson, P. 2001. Individual Differences and Instructed Language Learning. Amsterdam/ Philadelphie : John Benjamins

Vigotsky, L. S. 1997. Pensée et langage. Paris : La Dispute/SNEDIT.

\section{ANNEXES}

\section{Annexe 1}

Un exemple sélectif de résultats statistiques obtenus pour la validation d'un dispositif innovant en L2

Recherche-action L2 LEA : bilan juin 2007

7 MCF et 1 PRAG

1 étudiante de M1

275 étudiants

\section{Résultats comparatifs à l'examen}

Statistiques

\begin{tabular}{|l|c|c|c|c|c|}
\hline & Présents & Moyenne & Note max. & Note min. & Écart-type \\
\hline $2005 / 2006$ & 241 & 7,83 & 17,5 & 0 & 4,04 \\
\hline $2006 / 2007$ S1 & 275 & 9,27 & 17 & 1,5 & 3,71 \\
$2006 / 2007$ S2 & 271 & 9,37 & 18,5 & 1,5 & 3,14 \\
\hline
\end{tabular}

\section{Répartition des notes}

\begin{tabular}{|l|c|c|c|c|c|c|}
\hline & 15 ou + & $13-14$ & $10-12$ & $8-9$ & $7-6$ & 5 ou - \\
\hline $2005 / 2006$ & 8 & 14 & 74 & 66 & 37 & 42 \\
\hline $2006 / 2007$ S1 & 15 & 43 & 95 & 53 & 44 & 25 \\
$2006 / 2007$ S2 & 9 & 44 & 83 & 59 & 46 & 30 \\
\hline
\end{tabular}

Montrent un gain de 1,44 point (1,54 au S2) de moyenne, et un écart-type réduit : moins de notes aux extrêmes.

- Les étudiants sont globalement assez satisfaits du dispositif, avec un effet très net => la 
satisfaction est inversement proportionnelle à la taille des groupes.

- Les notes les plus basses concernent leur sentiment de progression au fil des cours, et les résultats à l'examen, paradoxalement, puisqu'ils sont meilleurs.

$\Rightarrow$ Difficulté de modifier les représentations.

Très peu de commentaires.

Annexe 2

Proposition de programme pour langue de spécialité et traduction en LEA

Diplôme : Licence

Intitulé : Langue de spécialité et traduction

\section{Organisation}

La formation proposée en langue de spécialité et traduction en LEA combine les cours en présence de l'enseignant et l'utilisation de la plate-forme d'enseignement Madoc. Les étudiants disposent sur la plate-forme d'un espace de cours, où ils trouveront les documents mis à leur disposition par les enseignants, ainsi que les tâches qu'ils auront à réaliser selon un calendrier défini. Ils bénéficieront d'un suivi personnalisé. Ils y trouveront également un module d'autoformation où ils pourront trouver des ressources pour améliorer leur niveau de compétence. Ils rencontreront leur enseignant au moins une fois par semaine par groupe de 12 étudiants.

L'organisation proposée permettra de personnaliser les relations avec les enseignants, et d'individualiser le travail étudiant. Parce qu'elle renforce la pratique orale et écrite, l'efficacité de la formation sera accrue. Enfin, avec l'objectif à terme de faciliter l'insertion professionnelle, la responsabilisation, l'autonomie, le travail en équipe, et la maîtrise des TIC sont autant d'atouts que les étudiants pourront développer.

\section{Compétences visées}

L'objectif de cette formation en anglais des affaires est de permettre aux étudiants de construire progressivement des compétences linguistiques (terminologie, discours et genre), communicationnelles, culturelles et méthodologiques, à l'écrit et à l'oral, liées aux situations qu'ils rencontreront dans le cadre des métiers des affaires et du commerce international. Les étudiants seront sensibilisés graduellement aux enjeux socioculturels et sociolinguistiques des problèmes de traduction dans leurs aspects linguistiques, techniques et sociaux. La spécialisation deviendra croissante à mesure de l'avancée dans le cursus.

Objectif final: donner aux étudiants les connaissances et les outils nécessaires pour qu'ils abordent avec confiance leur insertion professionnelle.

\section{Contenus}

La formation s'organise autour de quatre thématiques semestrielles (Tableau 1).

Tableau 1. Quatre thématiques semestrielles

\begin{tabular}{|l|l|}
\hline Thématiques L1 Sem 1 & Thématiques L1 Sem 2 \\
\hline Companies & Outsourcing \\
\hline Strategy & Counterfeiting \\
\hline \hline Development & Markets \\
\hline
\end{tabular}




\begin{tabular}{|l|l|}
\hline Marketing & Communication \\
\hline Thématiques L2 Sem 1 & Thématiques L2 Sem 2 \\
\hline Alliances & Technology \\
\hline Projects & Advertising \\
\hline Teamworking & Law \\
\hline Information & Brands \\
\hline Thématiques L3 Sem 1 & Thématiques L3 Sem 2 \\
\hline Investment & Banking \\
\hline Energy & Training \\
\hline \hline Going public & Consulting \\
\hline Competition & Recruitment \\
\hline
\end{tabular}

Autour de ces thématiques, les étudiants seront conduits à réaliser huit tâches par semestre en vue de la mise en place et du développement des compétences visées.

Communication à l'écrit : rédiger un courrier électronique, un fax, une lettre commerciale, un CV ou une lettre de motivation.

Rédiger un rapport, un mémorandum, un compte-rendu de réunion, un communiqué de presse, effectuer une recherche documentaire et rédiger la synthèse.

Passage d'une langue à l'autre sous forme de : synthèse/résumé/compte-rendu/ reformulation/traduction.

Communication à l'oral : Communication ou présentation orale (avec support)/ conversation téléphonique/entretien d'embauche/réunion/négociation.

Passage d'une langue à l'autre : interpréter, rendre compte à l'oral dans une autre langue d'une documentation écrite.

Autres compétences : utiliser Internet pour la traduction et pour la documentation.

\section{Validation}

$50 \%$ CC $+50 \%$ examen

Principe du contrat pédagogique : 8 tâches (et présence lors des regroupements et participation). Une tâche n'est pas considérée comme accomplie si l'autocorrection n'a pas été faite.

Principe de la validation d'un niveau individuel : un examen terminal écrit de 3 heures

\section{Documents supports}

En L1 : utilisation de Intelligent Business Intermediate. Pearson Longman et The Economist. En L2 et L3 : utilisation de Intelligent Business Upper Intermediate. Pearson Longman et The Economist

D’autres documents seront disponibles sur la plate-forme et/ou distribués par les enseignants. 


\section{NOTES}

1. LANSAD: Langues pour spécialistes d'autres disciplines; LLCE: Langue, littérature et civilisation étrangères.

2. Il existe un consensus dans la plupart des domaines de recherche aujourd'hui pour postuler qu'il n'existe pas de recherche objective, car la personnalité du chercheur oriente les choix méthodologiques et théoriques effectués. Il est donc nécessaire de "réintroduire le chercheur dans sa recherche ", c'est-à-dire de prendre en compte son positionnement pour relativiser ses conclusions. Les références sur ce point sont abondantes. Nous avons sélectionné Morin et Elias qui nous ont semblé les plus connus sur ce positionnement.

3. Les postes offerts en LANSAD et en LEA sont supérieurs au nombre de personnels qualifiés pour y postuler, comme le montrent année après année les chiffres publiés par la SAES et les dossiers à traiter en commission de spécialistes. En conséquence, nombre de littéraires sont nommés en LEA alors qu'ils n'ont pas les compétences en langue de spécialité. Le rôle du responsable de la filière est alors de leur offrir un dispositif suffisamment flexible et un accompagnement pour que leur acculturation soit possible.

\section{RÉSUMÉS}

Si l'anglais de spécialité est par définition la langue enseignée en LEA, le contexte dans lequel cet enseignement intervient présente des différences notables et impose une transposition didactique des recherches en langue de spécialité qui tienne compte non seulement des contraintes institutionnelles, mais également des paramètres socio-économiques qui conditionnent le recrutement dans la filière et les débouchés potentiels. À partir des résultats de six ans de travail de terrain en LEA, cet article se propose de montrer comment la rechercheaction accompagnée d'une réflexion sur le contexte d'enseignement, le public et les objectifs visés, les contenus d'enseignement, les tâches et les dispositifs a permis la mise en place d'une structure collaborative et de réduire la distance qui séparait savoirs théoriques et représentations des différents acteurs dans le souci d'accroître l'efficacité de la formation proposée. Il montrera comment la crédibilité et le rayonnement de cette formation peuvent être liés à ce type d'action. L'auteur se propose de développer l'application en LEA de la recherche en anglais de spécialité et de fédérer les énergies de ceux qui sont prêts à mettre en place une dynamique d'échanges.

ESP is what the applied languages section at the university is concerned with, and to that extent many similarities can be found with other university departments whose focus is not primarily on language itself. However, the context in which language teaching and learning take place is significantly different and thus ESP research is not directly transferable to this environment without taking into account the institutional and socio-economic parameters that are linked to students' reasons for applying for this course and the future career they may expect after graduation. Six years of teaching in this field have put the author in a position where she can show how a combination of action-research on the one hand and, on the other, an analysis of the teaching context, contents and objectives, learner profiles, and finally task-based instruction and 
distance-learning systems, have all enabled the implementation of a collaborative structure. It has also been possible to some extent to bridge the gap between what theory tells us about language learning and learners' and teachers' beliefs. This should improve the efficiency of the proposed learning system as well as the reliability and attractiveness of the course.

\section{INDEX}

Mots-clés : contexte, didactique, dispositif, langue de spécialité, LEA, recherche Keywords : applied languages, context, didactics, ESP, research, system

\section{AUTEUR}

\section{MARIE-FRANÇOISE NARCY-COMBES}

Marie-Françoise Narcy-Combes est Professeur à l'Université de Nantes où elle est chargée de la coordination de l'enseignement de l'anglais en LEA. Elle assure des enseignements en anglais des affaires dans cette filière et elle est aussi impliquée dans les enseignements en didactique des langues et des cultures pour la formation initiale et continue des enseignants dans ce domaine. Elle est l'auteur de Précis de didactique des langues (Ellipses 2005) et de La communication interculturelle en anglais des affaires (Presses Universitaires de Rennes 2006). mariefrancoise.narcy-combes@univ-nantes.fr 